\title{
Qualitative Analysis of Spray Characteristics of Impinging Jets Using a Gelled Non-Newtonian Propellant Simulant
}

\author{
Ali Darvan ${ }^{1}$, Azadeh Kebriaee ${ }^{1}$, Ali Nouri-Borujerdi ${ }^{1}$, Amir Nourian*2 \\ ${ }^{1}$ Sharif University of Technology, Tehran, Iran \\ ${ }^{2}$ Spray Research Group (SRG), School of Science, Engineering and Environment (SEE), \\ University of Salford, Manchester, UK \\ *Corresponding author email: ali.darvan.amiri@shairf.edu
}

\begin{abstract}
The utilization of liquid and solid fuels for propulsion and combustion processes with Newtonian characteristics are widely known. However, recent studies are considering the application of shear-thinning non-Newtonian fuels as alternative simulant since they have some advantages compared to the conventional propellants although there are challenges of providing better spray performance during the corresponding process. This paper, therefore, presents the results of the experimental investigations of the "near-field" spray characteristics with utilizing imaging techniques which evaluates the sheet formation and breakup length of four different spray patterns produced by the jet impingement of a gelled non-Newtonian propellant simulant. The qualitative analysis of this study shows that the spray patterns are different compared to those that are shaped by using Newtonian liquid fuels. This could lead to supposition that the non-Newtonian rheology of the gelled propellant simulant postponed the sheet and ligaments breakup, including the mode change of the atomization. In addition, the atomization of the sheet at different flow parameters could occur due to the formation and the sheet wave instability when aerodynamic and hydrodynamic of their origin are closely considered. This was further supported by the occurrence of perforations in the sheet.
\end{abstract}

\section{Keywords}

non-Newtonian liquid, impinging jet injectors, gelled propellant, near-field spray, sheet breakup.

\section{Introduction}

Advancement in aerospace industries and recently in space exploration technologies requires vehicles which are reliable, safe, and operable. Therefore, there is desirable to improve the performance and capability of these technologies as well as reducing their costs [1]. Liquid propulsion fuel system has a high demand in air transport industry since it has advantages of high specific impulse, trust management, hypergolicity, proper safety and the controllability through throttling. However, the main disadvantages of this type of fuel is the hazards which are related to the potential leakage of propellant [2]. This is especially true for hypergolic propellant combinations that combust upon contact between fuel and oxidizer without need for an external energy source. Moreover, the hypergolic propellant combinations are toxic and suspected carcinogen and the health hazards associated with these propellants have led to the strict exposure limits for personnel [3]. Therefore, in order to overcome the disadvantages of liquid propellants, there has been a renewed interest in gelled propellants over the past two decades.

The gelled propellants are formed by adding a gelling agent to a liquid fuel and they are stabilized mainly through physical interactions between polymer molecules and/or particles, known as the gelling agent, and the fuel [3]. The gelled propellant system considerably 
reduces the amount of vapor produced by the above-mentioned toxic hypergolic propellants, thereby reducing the risk of exposure. It also has a higher density compared to its liquid counterparts in which the storage containers that hold them can be lighter [4]. Combining the best characteristics of solid and liquid propellants is another benefit of gelled propellants as they can be stored like a solid fuel and flow like a liquid when they are under pressure. More importantly, by using gelled propellant, the risk of leaks and spills will be reduced which is beneficial due to the spontaneous ignition of hypergolic propellants upon contact. Despite the aforementioned advantages, the mixture of gelling agents and the liquid propellants, which mainly behaves Newtonian, is non-Newtonian fluids in which the increased apparent viscosity at shear levels of fuel injection systems is the major disadvantage and it makes the atomisation more difficult with a great pressure loss within the supply system. Therefore, the gelled propellants have to demonstrate shear-thinning behaviour in which the viscosity decreases with increasing shear rate [5]. Besides, the effect of gelled propellants on atomization is significant and this has been re-enforced by previous studies which showed the physical interactions of gelling agents affect the physical characteristics of the fuel and oxidizer (i.e., density, viscosity, surface tension). This, in turn, affects the flow, performance, injection, atomization, and the combustion [3].

The present paper demonstrates the effect of changing in the physical properties of the propellants by adding gelling agent on flow, spray and atomisation process, combustion behaviour, and, vitally important, selection of proper design of the injectors and combustion chamber systems to achieve the efficient combustion. The impinging jet injector was selected to be used in this study due to its simplicity in fabrication, low manufacturing costs as well as fine atomization and mixing of two streams of fuel and oxidizer [ [6]. In this injector a jet of fuel impinges a jet of oxidizer to atomize and mix the propellants using the fluid momentum. As shown in Figure 1 at the impingement point of two equal fluid jets an expanding thin liquid sheet forms in the plane perpendicular to the momentum vectors of the two jets. This liquid sheet, under certain conditions, breaks up downstream into a series of ligaments and untimely droplets through various competing forces which acting on it. The structure of the liquid sheet, its spray pattern, the ligaments' size, and droplet velocities are dependent upon the jets' velocities (U-jet) and diameters (d), the injector length-to-diameter ratio (L/d), the impingement angle $(2 \theta)$, the pre-impingement length-to-diameter ratio $(x / d)$, the physical properties of the liquid as well as the surrounding environment [7].

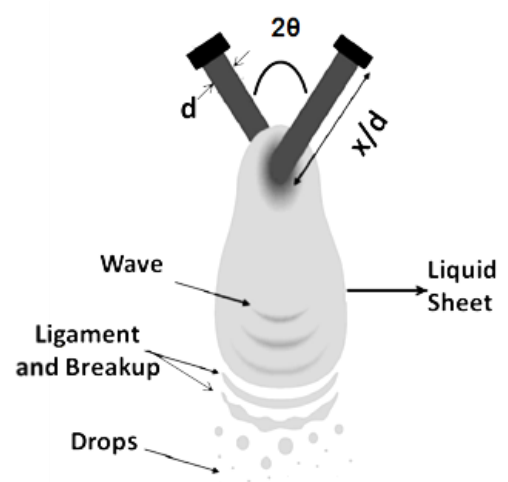

Figure 1: Typical impinging jet injector with its resulting flow

There is an abundance of available literature for spray and atomization characterizations of jet impingement with Newtonian liquids, However, only few studies have considered nonNewtonian substances and performed the same investigations and even less concerning the 
gelled propellants $[\underline{3}, \underline{8-11]}$. The objective of this study is therefore describing the mechanisms by which the non-Newtonian impinging jets are transformed into a sheet with waves on its surface, explaining how those waves affect sheet breakup and lead to formation of ligaments, and subsequently drops (primary atomization) and understanding of how the rheological behaviour of gelled non- Newtonian propellants influences each of these phenomena.

After all, since any change in geometry of injectors and experimental set-up/conditions can affect the characteristics of the generated spray and subsequent atomization, In this work, compared to previous research, we changed some of the effective features contributing to formation and characteristics of spray and atomization and used some novel combinations of Non-Newtonian material in terms of concentration as well as distinct pre-impingement length and impingement angle, and diameter and length for nozzles which are completely different from previous works.

\section{Experimental Setup}

The schematic diagram of the experimental setup for this study is shown in Figure 2. The liquid feeding system is a closed loop in which the liquid from a storage tank is driven to a pressurized vessel at a specific pressure using centrifugal pump. The liquid comes out from two stainless steel hypodermic needles with square ends and an internal diameter of 0.606 $\mathrm{mm}$. The impingement angle is fixed at 80 degrees using a rotary stage with the precision of \pm 1 degree. The pre-impingement length is also fixed about $5 \mathrm{~mm}$.

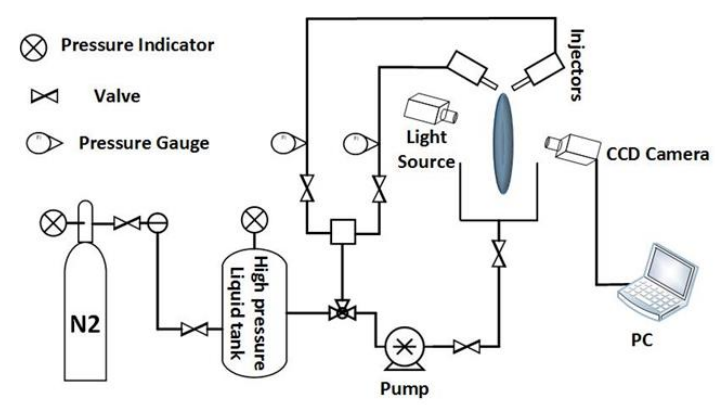

Figure 2: Schematic diagram of the experimental setup applied in current work.

Two pressure gauges were used to control the exit pressure of jets in order to investigate the impinging of two jets at different pressures and velocities. A shadowgraph imaging technique was used with setting the light source and the camera fixed in an axis normal to the liquid sheet. Within this setup the width and breakup length of the liquid sheet were captured from the front view. In order to demonstrate the gelled propellants with a behaviour of shear-thinning non-Newtonian fluid, a mixture of distilled water with 0.4 wt.\% Xanthan gum (XG) was used. In this mixture, distilled water acts as a Newtonian liquid and its mixture with $X G$ as a gelling agent changes the mixture to be a non-Newtonian fluid with density $(\rho)$ of $1050 \mathrm{~kg} / \mathrm{m}^{3}$. A rotational rheometer was also used to characterize rheological properties of the water-based gel simulant as shown in Figure 3. 


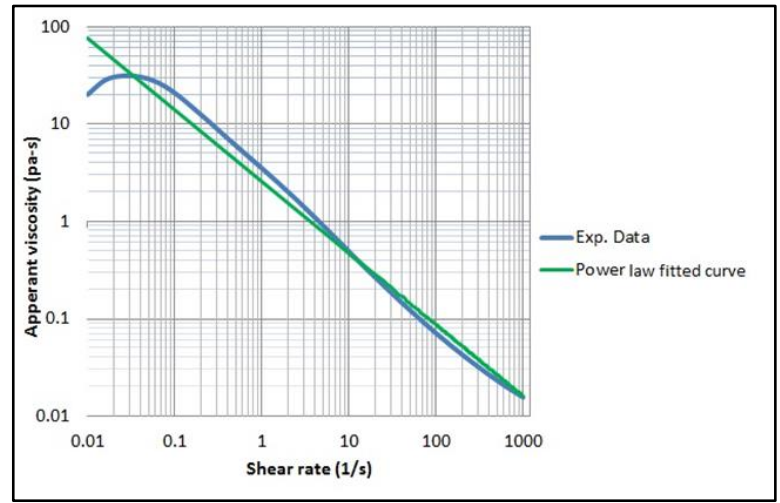

Figure 3: Shear viscosity as a function of shear rate

The rheological behaviour of the water-based gel simulant was described using the power-law equation within the measured range of shear rate as shown in Equation (1):

$$
\eta=K \gamma^{n-1}
$$

where $\eta$ and $\gamma$ denote the shear viscosity and shear rate, respectively. Also, $K$ is a consistency coefficient and $n$ is the flow index. By fitting the measured rheological curve to the power-law model, the value of $n$ and $K$ can be obtained as 2.57 and 0.265 , respectively. For analysing the Reynolds number in non-Newtonian fluids, the dependence of viscosity on the shear rate must be considered. In this study, a generalized Reynolds number $\left(\mathrm{Re}_{\mathrm{gen}}\right)$ based on the powerlaw model equation has been used. Equation 2 shows this model which was developed by Metzner and Reed [12].

$$
R e_{g e n}=\frac{\rho u^{2-n} d^{n}}{K\left(\frac{3 n+1}{4 n}\right)^{n} 8^{n-1}}
$$

\section{Results and Discussion}

This section presents the experimental results of the mixture of distilled water with 0.4 wt.\% $X G$ at different range of Reynolds number within ambient condition. Four different spray patterns have been identified throughout this investigation which will be described in the following sections.

\section{(1) Stable Closed Rim}

Figure 4 shows the image of the atomization at a very low Reynolds number. In this regime, an elliptical liquid sheet is formed at the impingement point of two jets and perpendicular to the jets' collision plane. 


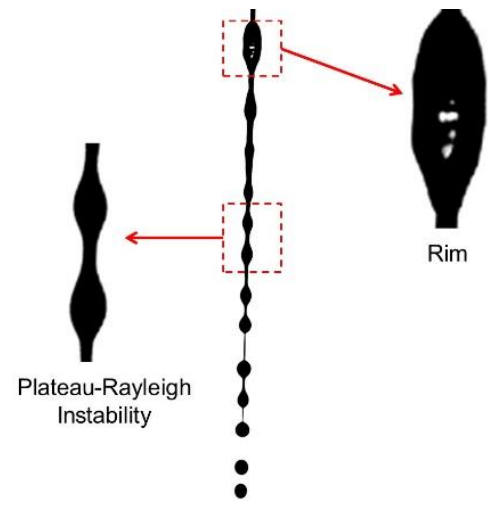

Figure 4: Image of a liquid sheet with $\operatorname{Re}=1377(\mathrm{U}-\mathrm{jet}=2.81 \mathrm{~m} / \mathrm{s})$

This liquid sheet is almost stable, however, at some point and due to the impact waves from the impingement point, it rippled. The sheet is surrounded completely by a distinct rim and since there is not sufficient momentum of jets to overcome the surface tension forces of the periphery of the sheet, the rim is thickened by rolling up of liquid on to itself. At the tip (downstream end) of the sheet the two arms of the rim finally converge under a distinct angle and forms a single jet stream which break up due to Plateau-Rayleigh type of instability and thus generate large droplets which are several times larger than the pre-impingement jet diameter. Contrary to the sheet formation from Newtonian liquids in which the inertia is balanced by the surface tension forces, in non-Newtonian liquids the viscosity also needs to be considered on the equilibrium of forces since it effects on the size and the shape of the liquid sheet.

\section{(2) Unstable closed rim, with the formation of vermicular ligaments}

At the middle range of Reynolds numbers, the spray pattern transforms to "unstable closed rim with the formation of vermicular ligaments" pattern as shown in Figure 5(a). In this regime, compared to the previous spray pattern, a larger, wider, and more cluttered liquid sheet appears. Also, more distinct disturbances are observed due to the start of the surface waves at the impact point of two jets. Although there are some instabilities on the sheet that cause instability, these do not appear to break due to the viscosity and surface tension forces. Hence, no ligament formation is observed from the liquid sheet at the downstream. This is, also, assumed that the inertial force is not capable of overcoming the viscous and surface tension forces on the sheet surface.
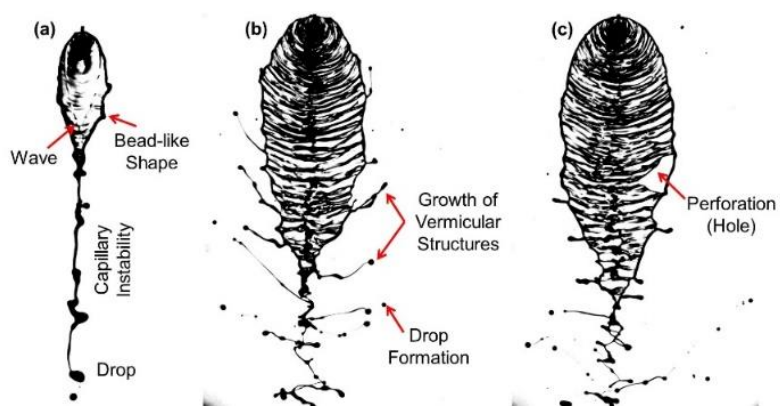

Figure 5: Images of a liquid sheet with (a) $R e=3524(\mathrm{U}$-jet $=4.83 \mathrm{~m} / \mathrm{s}),(\mathrm{b}$ and c) $\mathrm{Re}=8258(\mathrm{U}$-jet $=7.89 \mathrm{~m} / \mathrm{s})$

The expanding liquid, again, is collected at the boundary of the liquid sheet in the form of a relatively thick curved cylindrical rim under surface tension. The disturbances from the impingent point grow and spread downstream along the rim which becomes unstable under a capillary instability of the Plateau-Rayleigh type because of its cylindrical cross-section and a 
centrifugal Rayleigh-Taylor type of instability due to its curvature. When the low jets' velocity is slow, the beads cannot grow adequately before they reach to the tip point of the sheet. In this condition, the formation of droplets is due to the capillary instability of the irregular and distorted jet stream formed at the tip of the sheet. When the jets' exit velocity is increased the dimensions of the spray sheet increase and the disturbances on the sheet are intensified and spread throughout the entire sheet (see Figure 5(b)).

In addition, at higher velocities, disturbances at the edge of the sheet lead to the local momentum forces to be greater than the local surface tension force. This local imbalance causes some bead-like shapes to be produced at the rim of the sheet, which keep growing while moving along the edge. After growing to some extent, the beads develop and turn into drops attached to the rim via ligaments in a periodic manner which resemble a vermicular structure. These structures eventually become unstable and undergo Rayleigh breakup to form droplets on either side of the sheet. In this regime, when the inertia forces of impinging jets are high enough, the liquid sheet becomes significantly thin. This causes of forming perforation near the inner edge of the rim since the jets' momentum continues to spread it radially outward and impact waves make the thinned sheet unstable. Generally, the presence of perforations as shown in Figure 5(c), means that the liquid sheet is largely unstable and it is sufficiently thin.

\section{(3) Open rim with successive sinuous (bow) shaped ligaments}

Further increasing the inertial force leads to transition of morphology of the liquid sheet to become a "open rim with successive sinuous shaped ligaments" in which the liquid sheet is not entirely surrounded by a distinct rim and its lower part is opened. This transition is shown in Figure 6(a).

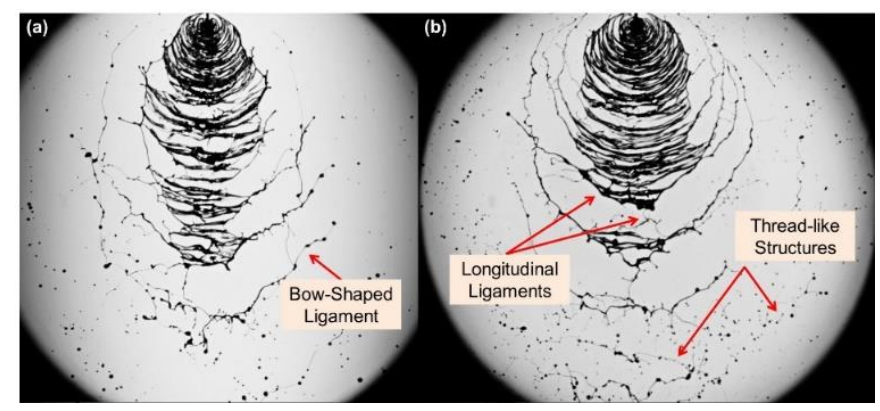

Figure 6: Images of a liquid sheet with (a) $R e=11986(\mathrm{U}$-jet $=9.78 \mathrm{~m} / \mathrm{s})$ and $(\mathrm{b}) \mathrm{Re}=16675(\mathrm{U}$-jet $=11.83 \mathrm{~m} / \mathrm{s})$

The sheet in this condition has a very wavy disturbed structure followed by a periodic separation of bow-shaped ligaments. Previous experiments have demonstrated that breakup of a liquid sheet formed by impinging jets generally occurs due to the formation of instability waves of aerodynamic or hydrodynamic origins. At a distinct higher velocity, the sheet is affected by impact waves as well as starts to be subjected to the aerodynamic instability which intensifies the generation of disturbances on both sides of the sheet. These disturbances are formed under the Kelvin-Helmholtz type instability when the relative velocity of the liquid sheet compared to the ambient medium is large enough to initiate and sustain unstable waves through shear stress [13]. Consequently, when the amplitude of the disturbing waves reaches to the critical values, the liquid sheet periodically begins to breakup into bow-shaped ligaments, mostly downstream of these ligaments decay into both drops and thread-like structures of different sizes (see Figure 6(b)). Moreover, as shown in Figure 7(a), further increasing of jets' velocities in this regime results in the frequency of formation of bow-shaped ligaments grow considerably as well as decreasing in the breakup length of sheet. 
Periodic separation of sinuous ligaments from the sheet is supported by the occurrence of perforations in the sheet. These perforations can expand and coalesce to generate ligaments of irregular shapes oriented in the direction of sheet displacement as shown in Figure 7(b). In addition to the bow-shaped ligaments, these longitudinally oriented ligaments, as shown in Figure 6(b), are a separate source of droplets in impingement atomization of the gelled liquids [14]. Although drops formation from the ligaments is observed, it appears that drops formation is hindered in comparison to the patterns of impinging water jets, as a Newtonian fluid, at the similar Reynolds numbers.

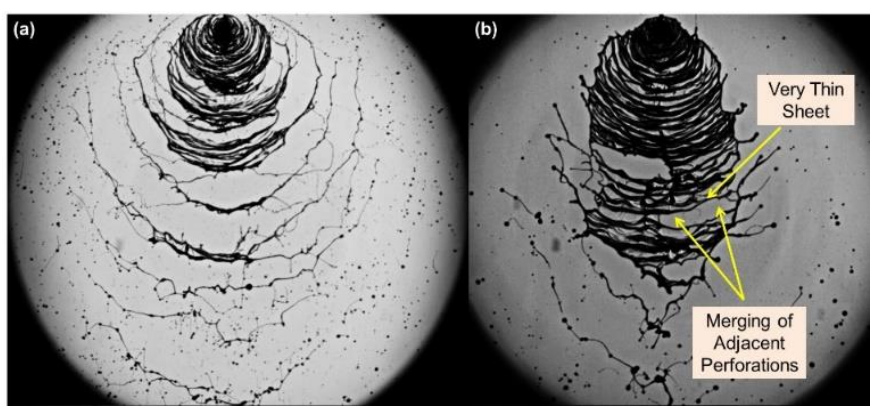

Figure 7: Images of a liquid sheet with (a) $R e=24797$

$(\mathrm{U}$-jet $=14.87 \mathrm{~m} / \mathrm{s})$ and $(\mathrm{b}) \operatorname{Re}=11986(\mathrm{U}$-jet $=9.78 \mathrm{~m} / \mathrm{s})$

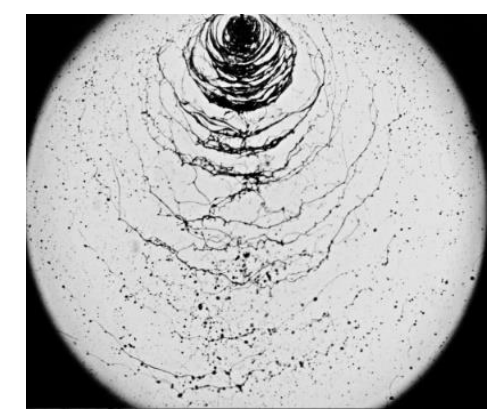

Figure 8: Image of liquid sheet with $\mathrm{Re}=41830$

(U-jet $=20.1 \mathrm{~m} / \mathrm{s}$ )

This is attributed to the dominance of viscous force as well as viscoelastic behaviour of the 0.4 wt.\% XG spray. Breakup of gelled liquids in this regime differs from Newtonian liquids due to presence of holes, their coalescence and the resulting longitudinally oriented ligaments.

\section{(4) Turbulent Regime}

Figure 8 presents the spray formation created in the extreme case of collision of two jets which is called "turbulent regime". In this regime a severely unstable small sheet is created in which the impact wave dominated over the whole sheet and can be recognized simply through the existence of elongated transverse structures.

The instabilities produced by the destructive inertial force led to the breakup of the liquid sheet periodically into ligaments which subsequently decay into droplets further downstream so that atomization occurs in all direction. Yet, there still exist thread-like structures as well as some parts of ligaments which remain unbroken in downstream, possibly because of the high velocity limitation of experimental setup used in the present work. The ligaments are expected to break completely into drops and to be thus entirely atomized by further increasing of the injection velocities. Compared to the foregoing regime, the distinct rim vanishes entirely in this regime and the breakup process becomes much more violent and begins earlier so that the sheet length is shortened. Furthermore, the sheet is torn azimuthally between two subsequent waves such that appearance of holes on the sheet is observed between two neighbouring waves.

\section{Conclusions}

Using shear-thinning non-Newtonian liquids as the gelled propellants attained renewed interest over the last two decades since they combine advantages of conventional liquid and solid propellants. As the main disadvantage for using non-Newtonian gelled propellants is production of quality atomization, further experimental studies for understanding of their spray and atomization characteristics are required. Near-field spray characteristics such as the sheet formation and breakup mechanism of the liquid sheet, produced by the like-on-like jet impingement of a gelled non-Newtonian propellant simulant, were experimentally investigated in this work using shadowgraph technic. Various spray patterns have been identified for the range of flow conditions including (i) stable closed rim; (ii) unstable closed rim with formation 
of worm like ligaments; (iii) open rim with successive bow shaped ligaments and (iv) turbulent regime. Some of these patterns were qualitatively different from those of Newtonian fluids so that non-Newtonian rheology of them is manifested as postponing of sheet and ligaments breakup as well as changing of atomization mode. The sheet atomization process for the range of flow parameters studied in the present work occurs due to the formation of instability waves of aerodynamic or hydrodynamic origins and is also supported by the occurrence of perforations in the sheet which are explained in detail.

\section{References}

[1] B. Palaszewski, L. S. lanovski, and P. Carrick, "Propellant technologies: far-reaching benefits for aeronautical and space-vehicle propulsion," Journal of Propulsion and Power, vol. 14, no. 5, pp. 641-648, 1998.

[2] W. J. Larson, G. N. Henry, and R. W. Humble, Space propulsion analysis and design. McGraw-Hill, 1995.

[3] J. A. Mallory, "Jet impingement and primary atomization of non-Newtonian liquids," Purdue University, 2012.

[4] G. Wilt, J. Hood, J. Jenkins, M. Thomas, J. Morris, M. Denn, B. Khusid, and Y. Shen, "Computational Design Tool for the Synthesis and Optimization of Gel Formulations (SOGeF)," CFD RESEARCH CORP HUNTSVILLE AL, 2009.

[5] P. Santos, R. Arnold, W. Anderson, M. Carignano, and O. Campanella, "Characterization of JP-8/SiO2 and RP-1/SiO2 gels," Engineering Letters, vol. 18, no. 1, pp. 41-48, 2010.

[6] G. Gill and W. Nurick, "Liquid rocket engine injectors," 1976.

[7] Y.-J. Choo and B.-S. Kang, "The velocity distribution of the liquid sheet formed by two low-speed impinging jets," Physics of fluids, vol. 14, no. 2, pp. 622-627, 2002.

[8] J. G. Lee, S. Fakhri, and R. Yetter, "Atomization and spray characteristics of gelledpropellant simulants formed by two impinging jets," in 45th AIAA/ASME/SAE/ASEE Joint Propulsion Conference \& Exhibit, 2009, p. 5241.

[9] N. S. Rodrigues, "Impinging jet spray formation using non-Newtonian liquids," 2014.

[10] N. S. Rodrigues, V. Kulkarni, J. Gao, J. Chen, and P. E. Sojka, "Spray formation and atomization characteristics of non-Newtonian impinging jets at high Carreau numbers," International Journal of Multiphase Flow, vol. 106, pp. 280-295, 2018.

[11] J. von Kampen, K. Madlener, and H. Ciezki, "Characteristic flow and spray properties of gelled fuels with regard to the impinging jet injector type," in 42nd AIAA/ASME/SAE/ASEE Joint Propulsion Conference \& Exhibit, 2006, p. 4573.

[12] A. Metzner and J. Reed, "Flow of non-newtonian fluids-correlation of the laminar, transition, and turbulent-flow regions," Aiche journal, vol. 1, no. 4, pp. 434-440, 1955.

[13] R. Li and N. Ashgriz, "Characteristics of liquid sheets formed by two impinging jets," Physics of fluids, vol. 18, no. 8, p. 087104, 2006.

[14] S. Y. Jejurkar, G. Yadav, and D. Mishra, "Characterization of impinging jet sprays of gelled propellants loaded with nanoparticles in the impact wave regime," Fuel, vol. 228, pp. 10-22, 2018. 